\section{Surgery for acute mediastinitis in oesophageal perforations and descending necrotising infection: complications, mortality and protective factors}

\author{
Jon Zabaleta, Borja Aguinagalde, \\ Marta G. Fuentes, Nerea Bazterargui, \\ Stephany M. Laguna, José M. Izquierdo \\ Thoracic Sugery Service, Hospital \\ Donostia, San Sebastian, Gipuzkoa, Spain
}

\begin{abstract}
Acute mediastinitis is a life-threatening situation that is associated with high rates of morbidity and mortality. The most common causes of mediastinitis are: oesophageal perforation, acute necrotising mediastinitis (ANM) and post-sternotomy mediastinitis. The aim of this study was to analyze prognostic factors and the differences between mediastinitis from various origins. A retrospective analysis was carried out on all patients operated on for acute mediastinitis between 2000 and 2009 . Surgical interventions were performed on 33 patients ( $69.7 \%$ male), the majority as a consequence of oesophageal perforation (21 cases). The overall mortality rate was $30 \%$. Better survival was seen in subjects less than 60 years of age, subjects with no comorbidities, and subjects who had undergone early tracheotomy. Patients with ANM were younger, had less comorbidities, diagnosed later and required more repeat interventions compared to those patients who had mediastinitis caused by oesophageal perforations. Mortality was lower in the ANM group, but this was not statistically significant. Protective factors in mediastinitis are: early diagnostic and treatment, age under 60 years, absence of major comorbidity and early tracheotomy.
\end{abstract}

\section{Introduction}

Mediastinitis refers to acute inflammatory processes of the mediastinal connective tissues. Generally the acute processes are due to gram-positive bacterial infections that produce purulent collections. ${ }^{1}$ Acute mediastinitis is a life-threatening situation that is associated with high morbidity and mortality rates. It may sometimes be hard to diagnose and manage, since its clinical presentation is non-specific and similar to other systemic infections or septic conditions. The mortality rates described in the literature vary between 14 and $42 \%{ }^{2}$
The most common causes of acute mediastinitis are oesophageal pathology, when the contents of the oesophagus pass through the mediastinum (oesophageal perforation), postoperative infections following a sternotomy and the descent of buccopharyngeal infections (acute necrotising mediastinitis or acute descending mediastinitis). ${ }^{3}$ Mediastinitis may be classified into three types (Endo's classification), depending on the extent of the disease. ${ }^{4}$

i) Type I: infection located above the carina.

ii) Type II: infection extended below the carina.

-Ila: anteroinferior location.

-IIb: posteroinferior location.

Depending on the type of mediastinitis, the surgical approach may differ. For type I, cervicotomy with drainage of the mediastinum may be sufficient, ${ }^{5}$ while a more aggressive approach is required for type II, involving posterolateral thoracotomy, sternotomy or videoassisted thoracoscopic surgery (VATS) ${ }^{6,7}$ A retrospective analysis was carried out on patients operated on for acute mediastinitis, with the aim of identifying factors that may affect survival and to analyze the differences in type II mediastinitis depending on the origin.

\section{Materials and Methods}

A retrospectively analysis was performed on all patients who underwent urgent surgical interventions for a diagnosis of acute mediastinitis at the study hospital between $1^{\text {st }}$ January 2000 and $31^{\text {st }}$ December 2009. In all cases a cervicothoracic Computer Tomography (CT) scan was used to obtain a diagnosis and as soon as this was confirmed, broad-spectrum antibiotic therapy was initiated, being replaced when appropriate, by specific antibiotics depending on the results obtained from an analysis of the evacuated pus and the debrided material. Demographic and etiological data were gathered retrospectively, as well as length of hospital and ICU stays, complications and mortality. The complications were classified according to the criteria of Clavien and Dindo ${ }^{8}$ (Table 1). Data were analysed using the statistical programme SPSS 15.0. For the statistical analysis, the Chi square test was used for comparing two categorical variables and nonparametric tests (Mann Whitney U test) for comparing one continuous variable and one dichotomous variable. Our institutional review board approved this study.

\section{Results}

During the study period, 33 patients under-
Correspondence: Jon Zabaleta Jiménez , Servicio de Cirugía Torácica, Hospital Donostia, San Sebastian, Paseo dr Beguiristain s/n. 20014 San Sebastian, Gipuzkoa, Spain.

Tel. +34.943.00.70.22 - Fax: +34.943 .00 .71 .44$

E-mail: jon.zabaletajimenez@osakidetza.net

Key words: mediastinitis, acute mediastinitis, acute necrotising mediastinitis, oesophageal perforation, thoracic surgery, mortality.

Acknowledgments: the authors would like to thank Nieves San Juan, by her assistance with the bibliographic searches.

Received for publication: 25 August 2011. Accepted for publication: 19 October 2011.

This work is licensed under a Creative Commons Attribution NonCommercial 3.0 License (CC BYNC 3.0).

(C) Copyright J. Zabaleta et al., 2011

Licensee PAGEPress, Italy

Chest Disease Reports 2011; 1:e15

doi:10.4081/cdr.2011.e15

went surgical intervention for acute mediastinitis: 23 men (69.7\%) and 10 women (30.3\%) with a mean age of 55.2 years (median 29.8 years, range 4-84 years). With regard to cause, 9 patients (27.3\%) met the Estrera criteria ${ }^{3}$ to be categorised as acute necrotizing mediastinitis (ANM), 21 cases (63.6\%) as oesophageal perforation, $1(3 \%)$ as spontaneous mediastinitis and $2(6.1 \%)$ as mediastinitis following scheduled oesophageal surgery. In the present series there were no patients with mediastinitis as a complication following cardiac surgery, since the speciality does not exist in the study hospital and patients suffering complications from cardiac surgery are admitted to the hospital where the initial surgery was carried out.

The average hospital stay was 29 days (median 26 days) and the median ICU stay was 20 days (median 22). Complications were observed in 27 patients (75.8\%), and we classified them using Clavien-Dindo International classification (Table 1): ${ }^{8} 1$ patient $(3.0 \%)$ presented grade I complications, 7 (21.2\%) grade II, 5 (15.2\%) grade IIIa, 3 (9.1\%) grade IIIb, 0 $(0.0 \%)$ grade IVa, $1(3.0 \%)$ grade IVb and 10 (30.3\%) grade V. Re-intervention was necessary in 8 cases (24.2\%) and 10 patients died (30.3\%). Septic shock (2 patients), multiple organ failure (FM0) (5 patients) and the respiratory distress (3 patients) were the leading causes of death.

In all cases, posterolateral thoracotomy was carried out in order to clean and debride the mediastinum with tube drainage being set up subsequently for the chest and mediastinum. In the initial procedure, in addition to the tho- 
racotomy, cervicotomy was performed in 8 cases (24.2\%), tracheotomy in 6 cases (18.8\%), gastrostomy in 20 cases (60.6\%) and jejunostomy in 13 cases (39.4\%).

An analysis of the patient's medical histories (Table 2) reveals that 5 had major comorbidity, 9 had minor comorbidity and 15 did not have any previous medical or surgical history. The mortality for the major comorbidity group was $100 \%$, for the minor comorbidity group $18.2 \%$ and for those with no previous history 6.6\%. Comparing mortality for each group $(\mathrm{P}<0.001)$, it was observed that presence of major comorbidity was a predictive factor for mortality, with a relative risk of death of 8 for this group (95\% CI: 2.77-23.05).

In $51.5 \%$ of cases the diagnosis, and consequently the treatment, was made over $24 \mathrm{~h}$ later than the onset of symptoms. Comparing mortality according to time to diagnosis, no significant differences was seen between those who were diagnosed and treated during the first $24 \mathrm{~h}$ (mortality 18.8\%) and those who were treated after $24 \mathrm{~h}$ (mortality 41.2\%) $\mathrm{P}=0.161$; OR 3.03 (95\% CI: 0.62-14.78). Despite this not being statistically significant, the mortality rate for the late diagnosis group was double that of the early diagnosis group. Of those patients with late diagnosis, the ANM group has a significantly better chance of survival (mortality 12.5\%) than the oesophageal perforation group (mortality $62.5 \%)(\mathrm{P}=0.039)$.

The effect of the different variables on mortality is reflected in Table 3 , where it can be seen; in addition to the points listed above, that age and early tracheotomy have a protective effect on mortality. No statistically significant differences were revealed on comparing mortality according to gender, presence or absence of complications, cause of mediastinitis or need for repeat intervention.

\section{Analysis by subgroup}

When the patients were split into two groups, ANM and oesophageal perforation, the following results can be observed.

In the oesophageal perforation group, spontaneous oesophageal rupture or Boerhaave's syndrome was responsible for $57.1 \%$ of cases, followed by endoscopic perforation at $23.8 \%$ and perforation by foreign bodies at $3 \%$. Eighty-one percent of injuries occurred to previously healthy oesophagi and the time to diagnosis was greater than $24 \mathrm{~h}$ in $61.9 \%$ of cases. The mean age of patients was 61.3 years (median 60 ) and $71.4 \%$ were male. In this group, mortality was $38.1 \%$ (8 cases); 18 patients (85.7\%) had complications (Table 4) and repeat intervention was necessary in 3 cases (14.3\%). Posterolateral thoracotomy was carried out in all cases to clean and debride and the following interventions were carried out on the oesophagus: oesophageal exclusion in 16 cases (76.2\%), primary closure in 2 cases

Table 1. Clavien-Dindo classification of surgical complications.

Grade I Any deviation from the normal postoperative course without the need for pharmacological treatment except antiemetics, antipyretics, analgesics, diuretics, electrolytes, and physiotherapy.

This grade also includes wound infections opened at the bedside

Grade II Requiring pharmacological treatment with drugs other than such allowed for grade I complications Blood transfusions and total parenteral nutrition are also included

Grade IIIa Requiring surgical, endoscopic or radiological intervention without general anesthesia. Grade IIIb Requiring surgical, endoscopic or radiological intervention under general anesthesia.

Grade IVa Life-threatening complication (including CNS complications)* requiring IC/ICU management.

Single organ dysfunction (including dialysis)

Grade IVb Life-threatening complication (including CNS complications)* requiring IC/ICU management Multiorgan dysfunction

Grade V Death of a patient

*Brain hemorrhage, ischemic stroke, subarrachnoidal bleeding, but excluding transient ischemic attacks. CNS, central nervous system; IC, intermediate care; ICU, intensive care unit.

Table 2. Prior associated comorbidity.

\begin{tabular}{ll} 
Minor & Major \\
Arterial hypertension (3 patients) & $\begin{array}{l}\text { Ischemic/hypertensive heart disease } \\
\text { Oesophagitis (2 patients) }\end{array}$ \\
(2 patients) \\
\hline Hernioplasty (2 patients) & Peripheral vascular disease (1 patient) \\
\hline Sleep apnoea/hypopnoea syndrome (1 patient) & Diabetes mellitus (1 patient) \\
Hyperuricemia (1 patient) & Chronic kidney failure with dialysis (1 patient) \\
\hline
\end{tabular}

(9.5\%) and oesophagectomy in 2 (9.5\%). In one case no procedure on the oesophagus was carried out because no macroscopic lesions were found; cleaning and debriding of the mediastinum was therefore carried out alone. Gastrostomy was performed on 20 patients (95.2\%) and jejunostomy for feeding in 13 patients (61.9\%).

The mean time between surgical intervention and start of oral tolerance was 11.4 days (median 6.5). In 3 cases (18.8\% of oesophageal exclusions) endoscopy with dilatation was required as the oesophagus did not open spontaneously, although in no case was surgical intervention required due to a failure of the oesophagus to open. On discharge, a barium contrast x-ray was taken on the final 12 patients, with satisfactory oesophagogastric transit in $100 \%$ of cases.

With regard to acute descending mediastinitis, the mean age of the 9 patients was 39.4 years (median 35.00 ) and $66.7 \%$ were male. All met the Estrera ${ }^{3}$ criteria for classification as acute descending mediastinitis. Bacteriological analysis of these patients showed Streptococcus bacteria in $88.9 \%$ of cases: of this number, $37.5 \%$ were of the alpha haemolytic group (S. viridans) and $62.5 \%$ of the beta haemolytic group ( $S$. pyogenes). The location of the primary infection was as follows: 2 parapharyngeal abscesses, 2 peritonsil-
Table 3. Variables affecting mortality.

\begin{tabular}{lcc} 
Factor & P & $\begin{array}{c}\text { Odds ratio } \\
(95 \% \text { CI })\end{array}$ \\
Age (>60 years) & 0.011 & $8.5(1.45-49.53)$ \\
Comorbidity & 0.000 & $0.125(0.043-0.360)$ \\
\hline Tracheotomy & 0.027 & $0.062(0.458-0.852)$ \\
Gender & 0.25 & $0.39(0.075-2.043)$ \\
\hline Time & 0.338 & $2.200(0.431-11.219)$ \\
Complications & 0.109 & $1.563(1.165-2.097)$ \\
\hline Cause (OP/ANM) & 0.139 & $4.923(0.515-47.071)$ \\
Reintervention & 0.611 & $1.54(0.289-8.250)$ \\
\hline
\end{tabular}

OP, oesophageal perforation; ANM, Acute necrotising mediastinitis.

lar abscesses, 2 tonsillitis, 1 epiglottitis, 1 submental abscess, 1 molar abscess.

The mean hospital stay was 30.4 days (median 22 days) and the mean ICU stay was 18.3 days (median 21$)$. Only one case (11.1\%) was diagnosed less than $24 \mathrm{~h}$ after manifestation of symptoms and in 8 cases (88.9\%) thoracotomy was carried out for the purpose of debridement, together with a cervicotomy to clean the neck. Tracheotomy was carried out in 6 cases (66.7\%), and in 4 cases (44.4\%) patients required reintervention for repeated drainage (both cervicotomies and thoracotomies). Using the Clavien-Dindo classifica- 
tion, seven complications were observed (77.8\%) (1 (11.1\%) grade I, 1 (11.1\%) grade II, 1 (11.1\%) grade IIIa, $2(22.2 \%)$ grade IIIb, 0 $(0.0 \%)$ grade IVa, $1(11.1 \%)$ grade IVb and 1 (11.1\%) grade V) and 1 patient (11.1\%) died. None of the patients in the ANM had important comorbidities.

A comparative analysis between the two groups was carried out (Table 4), in which no statistically significant differences were observed in the number of complications or in mortality, although a higher mortality was recorded in the oesophageal perforations group compared to the ANM group (38.1\% versus $11.1 \%$ respectively). The mean age of patients with descending mediastinitis was lower than in the perforations group (33.4 compared to 61.3 years of age; $\mathrm{P}=0.03$ ) and the number of repeat interventions was greater in the ANM group $(\mathrm{P}<0.05)$, with a mean of 1.2 thoracotomies per patient and 1.4 cervicotomies per patient.

\section{Discussion}

Acute mediastinitis is an uncommon disease (the population of the area of influence of the study hospital for this pathology is approximately 950,000 inhabitants, from which an estimated annual incidence of 3.5 cases per million inhabitants can be calculated), the preferred treatment for which is surgery in order to drain the septic focus. Acute mediastinitis surgery has high morbidity and mortality rates9, which have fallen in recent years 10 , due to the use of broad-spectrum antibiotics and an aggressive surgical approach, among other things. Ridder carried out a systematic review2 of the literature, comparing mortality rates in articles published in 1999-2008, 19901998 and 1960-1989, which were 15.5\%, 22.9\% and $34.9 \%$ respectively.

In our study, posterolateral thoracotomy is carried out in order to drain the mediastinal cavity, although some recent published studies report a minimally invasive approach using videothoracoscopy ${ }^{6,9}$ in cases of ANM and even oesophageal perforations. In patients for whom the origin of the mediastinitis is buccocervical, the cervical approach with widereaching debridement appears to be crucial and in cases of oesophageal origin, it is essential to repair the oesophagus. There is no consensus on the best technique for repairing the oesophagus. Especially depending on the time passed between perforation and surgery; the most frequently used techniques are primary repair with or without plastic surgery, oesophagectomy and oesophageal exclusion. ${ }^{10,11}$ In our study, the oesophageal exclusion technique has been carried out since 1997 on those patients in whom the time between

Table 4. Comparison of oesophageal perforation and acute necrotising mediastinitis.

\begin{tabular}{lcccc} 
& Overall (33) & $\begin{array}{c}\text { Oesophageal } \\
\text { perforation (21) }\end{array}$ & ANM (9) & P \\
Mortality & $10(30.3 \%)$ & $8(38.1 \%)$ & $1(11.1 \%)$ & 0.139 \\
Complications & $27(75.8 \%)$ & $18(85.7 \%)$ & $7(77.8 \%)$ & 0.593 \\
\hline Comorbidity & $7(21 \%)$ & $6(28.6 \%)$ & $0(0 \%)$ & 0.073 \\
Reintervention & $8(24.2 \%)$ & $3(14.3 \%)$ & $4(44.5 \%)$ & 0.010 \\
\hline Hospital stay (days) & 29.80 & 29.23 & 30.42 & 0.894 \\
Age (in years) & 55.27 & 61.33 & 39.44 & 0.003 \\
Gender $\quad($ male/female) & $23 / 10(69.7 \% / 30.3 \%)$ & $15 / 6(71.4 \% / 28.6 \%)$ & $6 / 3(66.7 \% / 33.3 \%)$ & 0.794 \\
Early diagnosis $(<24 h)$ & $16(48.5 \%)$ & $13(61.9 \%)$ & $1(11.1 \%)$ & 0.011 \\
\hline
\end{tabular}

ANM, acute necrotising mediastinitis.

perforation and treatment is greater than $12 \mathrm{~h}$. In contrast to other groups, ${ }^{12}$ the authors consider oesophageal exclusion to be a safe, effective technique for treating oesophageal perforation and oesophagectomy is reserved for those patients who, in addition to the perforation, have another related oesophageal pathology. We made oesophageal exclusion with nonreabsorbible stapler in both sides of the perforation ( 7 cases) or superior to the perforation (9 cases). We don't perform any diversion technique and we wait for spontaneous recanalization of the oesophagus. The mean time between surgical intervention and start of oral tolerance was 11.4 days (median 6.5), that is similar to time needed to reintroduce oral ingestion in groups treated by other techniques. ${ }^{13}$ With regard to minimally invasive surgery, two recently published studies are of particular interest, given that they examine VATS as a treatment for ANM14 (with a mortality rate of $16.7 \%$ ), and for mediastinitis caused by oesophageal perforation, also performing the oesophageal repair using VATS. ${ }^{9}$ We don't have enough VATS experience for treat this urgent pathology with minimal invasion, but, in expert hands it can be a proper treatment with good results published. ${ }^{6,10}$

One of the factors influencing survival is the time passed between onset of symptoms and diagnosis, with late diagnosis being related to lower survival rates: $:^{13,15}$ in the present series it can be observed that patients diagnosed beyond the initial $24 \mathrm{~h}$ have half the chance of survival as those diagnosed before. This finding seems obvious if we consider that acute mediastinitis is a serious infection which, over time, leads to multiple organ failure as a result of sepsis, thus increasing the probability of death. Analysing the patients in the late diagnosis group, it can be observed that patients with oesophageal perforation have lower survival rates than patients with ANM, with the difference between the two groups being statistically significant $(\mathrm{P}=0.039)$. Time is a determining factor in mediastinitis, and that's why a cervicothoracic CT scan is carried out in as soon as mediastinitis is suspected. Based on the CT findings, different treatments are necessary: for type I cases, cervicotomy and mediastinal drainage through the incision ${ }^{4,5}$ or percutaneous drainage could be sufficient. For type II cases, even though there are groups that carry out sternotomy for cases of IIa, ${ }^{7,16}$ the authors believe that it is appropriate to perform posterolateral thoracotomy in all cases, since the entire mediastinal cavity may be drained via the thoracotomy and the risk of osteonecrosis following sternotomy is avoided. ${ }^{17}$ In our study, a right thoracotomy through the fifth intercostal space, azygous vein section and opening of the mediastinal pleura from the vertex to the base in order to expose the entire mediastinum was chosen as our first-line therapy. In those cases in which the CT scan shows left-side predominance or a left-sided pleural effusion, a left-sided thoracotomy was preferred over a right-sided thoracotomy. In these cases, the mediastinal approach may be complicated by the presence of the heart and the aorta.

The patients in the ANM group had a lower mean age and require more reinterventions than those in the perforations group. Many publications argue for repeat thoracotomy and repeat cervicotomy in order to clean and debride the mediastinum in those cases in which clinical progress and CT imaging suggests perstsitent collections. This active surgical approach is fundamental for eradicating the source of infection, which is why recent research reports high numbers of cleaning reinterventions which, together with an improvement in antibiotic treatments, has resulted in an improved survival rate for those patients. $^{2}$

Another controversial point is whether it is ideal to carry out a tracheotomy in those patients in whom intubation seems to be difficult, or who look likely to require mechanical ventilation for a long period of time. Tracheotomy was carried out in 6 patients (all 
from the ANM group) and was not performed in another 27. Of the 6 who received tracheotomy, none died, while 10 patients died from the other group (37.04\%) ( $\mathrm{P}=0.027)$. Comparing mortality in the ANM group between tracheotomy patients (0 cases) and non-tracheotomy patients (1 case, $33.33 \%$ ), no differences were seen. Five complications (83.3\%) were observed in the tracheotomy group and 22 (81.5\%) in the non-tracheotomy group (NS). There are currently authors who do not carry out tracheotomies because they consider that it facilitates the aspiration of purulent material and the dissemination of the infection to the anterior mediastinum, contributing to the persistence of cervical and mediastinal sepsis. ${ }^{3,17}$ In contrast, Gaudino et al. published a cardiac surgery review, ${ }^{18}$ in which 5095 operated patients were analysed in order to evaluate the incidence of mediastinitis and sternal infection in patients who had undergone post-operative tracheotomy. Of these 5095 patients, tracheotomy was carried out in 57 cases and mediastinitis did not occur in a single one; moreover, no increase in the incidence of cases of bacterial growth in the sternum was observed $(\mathrm{P}=0.61)$. In the light of these findings, together with the results from the present series, the authors would continue to recommend early tracheotomy in cases for which intubation seems problematic or which look likely to require mechanical ventilation for a long period of time.

The majority of patients were previously in good health and those patients with major comorbidities had a higher mortality rate. These findings coincide with those published by Deu-Martin ${ }^{3}$ in 2010, in which the presence of lung-related comorbidity and diabetes mellitus were associated with a worse prognosis and higher mortality rates. In our series, we didn't find any ANM patients with major comorbidities or any inmunodefficiency, and the median age was lower than the oesophageal perforation group. Comparing the mortality of patients over and under the age of 60 , the older group had a eight fold greater probability of dying (OR 8.5 ; 95\% CI: 1.48 49.53) $(\mathrm{P}=0.011)$.

\section{Conclusions}

The authors' preferred treatment strategy for acute mediastinitis in all suspected cases involves a cervicothoracic CT scan to confirm the diagnosis. Once confirmed, broad-spectrum antibiotic treatment is initiated and a surgical intervention is carried out, involving posterolateral thoracotomy for cleaning and debridement and cervicotomy if the origin is buccopharyngeal. Thoracic CT scans are carried out at intervals between 2 to 10 days after the operation, depending on the patient's progress, and debridement is repeated if cervical or mediastinal collections persist.

Protective factors in mediastinitis are: early diagnostic and treatment, age under 60 years, absence of major comorbidities and early tracheotomy.

\section{References}

1. de Freitas RP, Fahy CP, Brooker DS, et al. Descending necrotising mediastiniis: a safe treatment algorithm. Eur Arch Otorhinolaryngol 2010;264:181-7.

2. Ridder GJ, Maier W, Kinzer S, et al. Descending necrotizing mediastinitis: contemporary trends in etiology, diagnosis, management, and outcome. Ann Surg 2010;251:528-34.

3. Deu-Martin M, Saez-Barba M, Lopez S, et al. Mortality risk factors in descending necrotizing mediastinitis. Arch Bronconeumol 2010;46:182-7.

4. Uwa N, Terada T, Saeki N, et al. Clinical features of 5 patients with descending necrotizing mediastinitis originating from deep neck infection. Auris Nasus Larynx 2010;37:530-4.

5. Honguero Martinez AF, Arnau OA, Fernandez CA, et al. Descending necrotizing mediastinitis: treatment by transcervical thoracic drainage. Arch Bronconeumol 2005;41:293-94.

6. Shimizu K, Otani Y, Nakano T, et al. Successful video-assisted mediastinoscopic drainage of descending necrotizing mediastinitis. Ann Thorac Surg 2006;81: 2279-81.
7. Singhal P, Kejriwal N, Lin Z, et al. Optimal surgical management of descending necrotising mediastinitis: our experience and review of literature. Heart Lung Circ 2008;17:124-8.

8. Dindo D, Demartines N, Clavien PA. Classification of Surgical Complications. A New Proposal With Evaluation in a Cohort of 6336 Patients and Results of a Survey. Ann Surg 2004;240:205-13.

9. Cho JS, Kim YD, I H, et al. Treatment of mediastinitis using video-assisted thoracoscopic surgery. Eur J Cardiothorac Surg 2008;34:520-4.

10. Rohatgi A, Papanikitas J, Sutcliffe R, et al. The role of oesophageal diversion and exclusion in the management of oesophageal perforations. Int J Surg 2009; 7:142-4.

11. Sutcliffe RP, Forshaw MJ, Datta G, et al. Surgical management of Boerhaave's syndrome in a tertiary oesophagogastric centre. Ann Roy Coll Surg 2009;91:374-80.

12. Macri P, Jimenez MF, Novoa N, Varela G. A descriptive analysis of a series of patients diagnosed with acute mediastinitis. Arch Bronconeumol 2003;39:428-30.

13. Eroglu A, Can Kurkcuogu I, Karaoganogu $\mathrm{N}$, et al. Esophageal perforation: the importance of early diagnosis and primary repair. Dis Esophagus 2004;17:91-4.

14. Chen KC, Chen JS, Kuo SW, et al. Descending necrotizing mediastinitis: a 10 -year surgical experience in a single institution. J Thorac Cardiovasc Surg 2008;136:191-8.

15. Shaker H, Elsayed H, Whittle I, et al. The influence of the golden $24 \mathrm{~h}$ rule on the prognosis of oesophageal perforation in the modern era. Eur J Cardiothorac Surg 2010;38:216-22.

16. Karkas A, Chahine K, Schmerber S, et al. Optimal treatment of cervical necrotizing fasciitis associated with descending necrotizing mediastinitis. Br J Surg 2010; 97:609-15.

17. Hirai S, Hamanaka Y, Mitsui N, et al. Surgical treatment of virulent descending necrotizing mediastinitis. Ann Thorac Cardiovasc Surg 2004;10:34-8.

18. Gaudino M, Losasso G, Anselmi A, et al. Is early tracheostomy a risk factor for mediastinitis after median sternotomy? J Cardiac Surg 2009;24:632-6. 